\title{
Porphyromonas gingivalis ATCC 33277 promotes intercellular adhesion molecule-1 expression in endothelial cells and monocyte-endothelial cell adhesion through macrophage migration inhibitory factor
}

Wanyue $\mathrm{Xu}^{\dagger}$, Yaping Pan ${ }^{\dagger}$, Qiufang Xu, Yun Wu, Jiayu Pan, Jingya Hou, Li Lin, Xiaolin Tang, Chen Li, Jingbo Liu and Dongmei Zhang* (1)

\begin{abstract}
Background: Porphyromonas gingivalis (P. gingivalis), one of the main pathogenic bacteria involved in periodontitis, induces the expression of intercellular adhesion molecule - 1 (ICAM-1) and monocyte-endothelial cell adhesion. This effect plays a pivotal role in atherosclerosis development. Macrophage migration inhibitory factor (MIF) is a multifunctional cytokine and critically affects atherosclerosis pathogenesis. In this study, we tested the involvement of MIF in the P. gingivalis ATCC 33277-enhanced adhesive properties of endothelial cells.

Results: Endothelial MIF expression was enhanced by P. gingivalis ATCC 33277 infection. The MIF inhibitor ISO-1 inhibited ICAM-1 production in endothelial cells, and monocyte-endothelial cell adhesion was induced by $P$. gingivalis ATCC 33277 infection. However, the addition of exogenous human recombinant MIF to P. gingivalis ATCC 33277-infected endothelial cells facilitated monocyte recruitment by promoting ICAM-1 expression in endothelial cells.

Conclusions: These experiments revealed that MIF in endothelial cells participates in the pro-atherosclerotic lesion formation caused by P. gingivalis ATCC 33277 infection. Our novel findings identify a more detailed pathological role of $P$. gingivalis ATCC 33277 in atherosclerosis.
\end{abstract}

Keywords: Porphyromonas gingivalis, Macrophage migration inhibitory factor, Intercellular cell adhesion molecule-1, Endothelial cells

\section{Background}

Many epidemiological studies have associated severe forms of periodontitis with atherosclerosis [1]. Porphyromonas gingivalis (P. gingivalis), a Gram-negative oral anaerobe, has been identified as one of the main pathogenic bacteria in periodontitis [2]. The DNA of $P$. gingivalis has been found in coronary stenotic artery

\footnotetext{
*Correspondence: juliyia@163.com

${ }^{\dagger}$ Equal contributors

Department of Periodontics and Oral Biology, School of Stomatology, China Medical University, Nanjing North St.117, Shenyang, Liaoning 110002, China
}

plaques of myocardial infarction patients [3, 4]. Furthermore, animal experiments have shown that $P$. gingivalis infection directly induces and accelerates atherosclerotic lesion development in pigs and mice [5, 6]. In vivo studies have suggested that $P$. gingivalis enters the systemic circulation through inflammation-injured epithelial structures; then, this bacterium adheres to and invades vascular endothelial cells, proliferates in host cells, promotes the release of a variety of proinflammatory cytokines and induces atherosclerosis formation [7-11]. 
Macrophage migration inhibitory factor (MIF) has been recognized as a key factor in the vascular processes leading to atherosclerosis [12-14]. MIF expression in endothelial cells is dysregulated in response to proatherogenic stimuli during the development of atherosclerotic lesions in humans, rabbits, and mice $[15,16]$. Recent research showed that MIF increased monocyte recruitment during the process of atherosclerosis development [17]. One of the mechanisms of this effect is the MIF-mediated upregulation of adhesion molecule expression in vascular endothelial cells, which causes the monocytes flowing rapidly in blood circulation to decelerate, roll on the vessel wall, aggregate and adhere to the vessel wall [18].

Studies have shown that increased intercellular adhesion molecule -1 (ICAM-1) expression is one of the molecular mechanisms of the pathological changes during the early stage of atherosclerosis. By mediating leukocyte adhesion, ICAM-1 increased plaque instability and accelerated plaque rupture and thrombosis, resulting in cardiovascular disease (CVD) events [19].

Our previous studies have found that $P$. gingivalis infection increases ICAM-1 expression in endothelial cells and monocyte-endothelial cell adhesion [20]. These findings suggested that $P$. gingivalis induces the inflammatory process of atherosclerosis. However, the exact role that $P$. gingivalis plays in the development of atherosclerosis is still unclear. We hypothesized that $P$. gingivalis infection promotes the formation of atherosclerosis through MIF. In the present study, we examined the MIF production induced by $P$. gingivalis ATCC 33277 in endothelial cells. We also investigated the impact of MIF on the adhesive properties of endothelial cells pretreated with the antagonist ISO-1 or human recombinant MIF (rMIF) plus ISO-1. Our novel findings have identified a more detailed pathological role of $P$. gingivalis in atherosclerosis.

\section{Methods}

\section{Bacterial strains and culture methods}

The $P$. gingivalis strain ATCC 33277 was anaerobically $\left(80 \% \mathrm{~N}_{2}, 10 \% \mathrm{O}_{2}, 10 \% \mathrm{H}_{2}\right)$ cultured in brain heart infusion broth that contained defibrinated sheep's blood (5\%), hemin $(0.5 \%)$ and vitamin $\mathrm{K}(0.1 \%)$ at $37{ }^{\circ} \mathrm{C}$. Bacterial cells were cultured overnight until the optical density reached 1.0 at $600 \mathrm{~nm}$; then, the cells were resuspended in Dulbecco's modified Eagle medium (DMEM, Gibco BRL, Carlsbad, CA, USA) at a final concentration of $1 \times 10^{12}$ cells $/ \mathrm{L}$.

\section{Cell lines}

The human umbilical vein endothelial cell line EA.hy926 and the THP-1 monocyte model (a monocytic leukaemia cell line) were purchased from Keygen Biotech company (Nanjing, China). EA.hy926 cells were cultured in
DMEM containing $15 \%$ fetal bovine serum, and the THP-1 cells were cultured in DMEM containing 10\% fetal bovine serum at $37{ }^{\circ} \mathrm{C}$ in $5 \% \mathrm{CO}_{2}$. EA.hy926 cells $\left(10^{5}\right.$ cells $\left.\mathrm{mL}^{-1}\right)$ were seeded in the tissue plate wells and were cultured until a confluent monolayer formed for subsequent study. Cell viability, which was $>90 \%$ for all the infection assays, was determined by trypan blue exclusion assay. THP-1 cells were labeled with the fluorescent dye calcein AM $(0.1 \mathrm{mg} / \mathrm{mL}$; BioVision, CA, USA) for $30 \mathrm{~min}$ before being co-cultured with EA.hy926 cells.

\section{Enzyme linked immunosorbent assay (ELISA)}

Bacterial suspensions were added to the EA.hy926 cells at a multiplicity of infection (MOI) of 100 for 4, 10 or 24 h, while Escherichia coli (E. coli) lipopolysaccharide (LPS) (1 $\mu \mathrm{g} / \mathrm{mL}$; Cayman Chemical, Ann Arbor, MI, USA) was used as a positive control [21]. The MIF level was determined using ELISA kits (BD Biosciences, Mountain View, CA, USA). The optical density was measured at $450 \mathrm{~nm}$, and the MIF concentration was extrapolated from the standard curve according to the manufacturer's instructions.

\section{Western blot}

The EA.hy926 cells were pretreated with the MIF antagonist ISO-1 (25 $\mu \mathrm{M}$; Cayman Chemical) or human rMIF $(0.5 \mu \mathrm{g} / \mathrm{mL}$; Cayman Chemica) plus ISO-1 for $1 \mathrm{~h}$; then, the cells were infected with $P$. gingivalis ATCC 33277 at an MOI of 100 for $24 \mathrm{~h}$. The whole cell protein of EA.hy926 cells was extracted, and Western blotting was performed.

The EA.hy926 cells were lysed, and the protein concentration was determined by a BCA assay. Equal amounts of whole cell lysate were separated with $8 \%$ SDS-polyacrylamide gel electrophoresis and were transferred to a nitrocellulose filter membrane. After blocking, the protein was blotted with rabbit monoclonal anti-ICAM-1 antibody (1:500; Wanlei, Shenyang, China) and goat anti-rabbit Dylight 800-conjugated fluorescent antibody (1:1000; Abbkine Inc., Redlands, CA, USA). Western blot analysis was performed with Odyssey CLX (LI-COR, Lincoln, NE, USA).

\section{Quantitative real-time polymerase chain reaction (qRT- PCR)}

EA.hy926 cells were treated as mentioned above (in Western blot analysis). Then, the total RNA of EA.hy926 cells was extracted using TRIzol reagent (Invitrogen, Carlsbad, CA, USA). To remove the genomic DNA, total RNA was treated with DNase I for $2 \mathrm{~min}$ at $42{ }^{\circ} \mathrm{C}$ following the manufacturer's protocol. The RNA integrity was checked via electrophoresis on $1.0 \%$ agarose gels. The RNA purity was identified by the $260 / 280 \mathrm{~nm}$ 
optical density ratio, and RNA samples with an 260/ $280 \mathrm{~nm}$ optical density ratio greater than 1.9 were selected for later analysis. Next, cDNA was synthesized using a reverse transcription system (Vazyme, Beijing, China) [22].

qRT-PCR was performed using Biosystems 7500 Fast real-time PCR and SYBR Premix Ex Taq II (RR047, RR420, Takara, Tokyo, Japan) according to the manufacturer's instructions. Specific primers were 5'TCGGCACAAAAGCACTATATG -3' (forward), 5'ACAGGACAAGAGGACAAGGC-3' (reverse) for ICAM-1 and 5'-GAAGGTCGGAGTCAACGGAT-3' (forward) and 5' - CCTGGAAGATGGTGATGG GAT-3' (reverse) for Glyceraldehyde-3-phosphate dehydrogenase (GAPDH). Primers were designed with Primer Premier 5 software. The results are expressed as the relative ICAM-1 mRNA levels compared with the untreated control, which was considered $100 \%$.

\section{THP-1 adhesion to EA.hy926 cells}

EA.hy926 $\left(10^{5}\right.$ cells $\left.\mathrm{mL}^{-1}\right)$ were seeded on 6-well plates at $2 \times 10^{5}$ cells per well and were cultured to form a confluent monolayer. Then, the cells were pretreated with the MIF antagonist ISO-1 $(25 \mu \mathrm{M})$ or rMIF $(0.5 \mu \mathrm{g} / \mathrm{mL})$ plus ISO-1 for $1 \mathrm{~h}$. Next, the cells were infected with $P$. gingivalis ATCC 33277 at an MOI of 100 for $23 \mathrm{~h}$. Next, $1 \times 10^{6}$ THP- 1 cells were labelled with $5 \mu \mathrm{M}$ calcein-AM and were co-cultured with the EA.hy926 cells for another $1 \mathrm{~h}$. Non-adherent THP-1 cells were gently washed away with PBS twice. The adherent THP-1 cells remaining on the monolayer of endothelial cells were visualized using a fluorescence microscope (Nikon 80i, Tokyo, Japan); 3 fields under the microscope $(\times 100)$ were randomly selected; and the fluorescence-labeled THP-1 cells were assessed by cell counting assays [23].

All the experiments were performed in triplicate wells for each condition and repeated at least three times.

\section{Statistical analysis}

All data are presented as the means \pm SD of three independent experiments. Statistical analysis was performed using one-way ANOVA, and the Student-Newman-Keul test was applied to compare differences from each other group (SPSS 17.0 software, IBM). $P$-values $<0.05$ were considered statistically significant.

\section{Results}

P. gingivalis ATCC 33277 infection enhances MIF secretion in EA.hy926 cells

We evaluated the effect of $P$. gingivalis ATCC 33277 on MIF expression. E. coli-LPS was used as a positive control, since MIF release is induced by proinflammatory factors such as LPS [21, 24]. The ELISA results revealed that $P$. gingivalis ATCC 33277 infection significantly increased MIF secretion in EA.hy926 cells. Compared with the control level, MIF expression was increased 2.25-fold $(\mathrm{MOI}=100)$ by $P$. gingivalis ATCC 33277 infection for $24 \mathrm{~h}(P<0.01)$. P. gingivalis ATCC 33277 did not significantly affect MIF expression at the early time point, including 4 and $10 \mathrm{~h}$ (Fig. 1). P. gingivalis infection at an MOI of 100 for $24 \mathrm{~h}$ was chosen to evaluate the impact of MIF on the increased adhesive properties of endothelial cells in the following studies.

\section{P. gingivalis ATCC 33277 infection increase expression of ICAM-1 and ICAM-1 mRNA in EA.hy926 through MIF}

To determine the impact of MIF on ICAM-1 expression, the MIF antagonist ISO-1 and rMIF were used. The results revealed that $P$. gingivalis ATCC 33277 infection $(\mathrm{MOI}=100: 1,24 \mathrm{~h})$ induced a significant increase in ICAM-1 expression. We discovered that this inductive effect of $P$. gingivalis ATCC 33277 was blocked by the MIF antagonist ISO-1. $P$. gingivalis-induced ICAM-1 expression was significantly reduced (by $49.78 \%$ ) by ISO-1. Moreover, the inhibitory effect of ISO-1 was neutralized by exogenous rMIF. Sufficient exogenous rMIF supplementation rescued ICAM-1 expression. ICAM-1 expression was increased 1.95 -fold in the rMIF group compared to the ISO group (Fig. 2a and b).

These findings were further confirmed by the qRTPCR results, which detected the ICAM-1 gene transcription level under the same conditions as described above. The ICAM-1 mRNA level was also significantly reduced in ISO-1-treated cells, with an $81.97 \%$ reduction compared with that in P. gingivalis-infected cells. Similarly, exogenous rMIF increased the ICAM-1 mRNA level, which was 3.51-fold higher in the rMIF group compared with the ISO group (Fig. 2c).

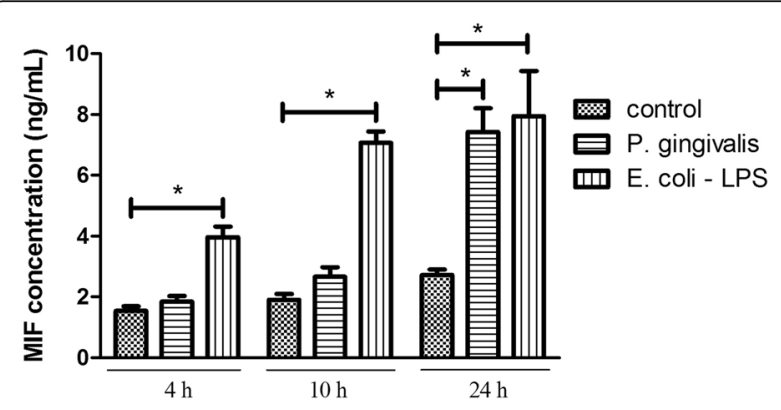

Fig. 1 Porphyromonas gingivalis (P. gingivalis) ATCC 33277 infection enhances MIF secretion in EA.hy926 cells. EA.hy926 were challenged with Escherichia coli (E. coli) lipopolysaccharide (LPS, $1 \mu \mathrm{g} / \mathrm{mL}$ ) or $P$. gingivalis $(\mathrm{MOI}=100)$ for 4,10 or $24 \mathrm{~h}$. MIF levels was analyzed using ELISA. Infection of $P$. gingivalis significantly increased MIF secretion in EA.hy 926 cells. EA.hy 926 were incubated with medium only as a control. E.coli LPS was used as a positive control. The I bar shows the standard deviation. ${ }^{*} P<0.01$ 


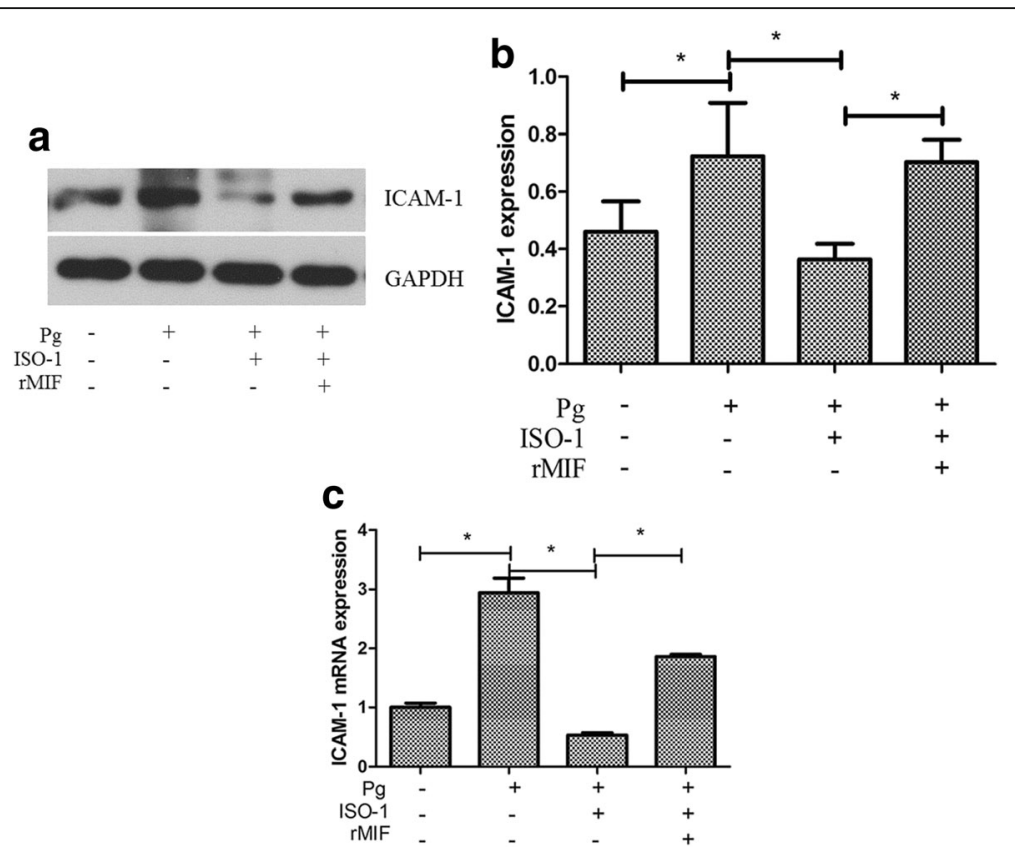

Fig. 2 Macrophage migration-inhibitory factor (MIF) acted as a regulator in Porphyromonas gingivalis (P. gingivalis)-induced intercellular adhesion molecule-1 (ICAM-1) expression. a Western blot analysis of ICAM-1 in EA.hy926 cells. $\mathbf{b}$ Quantitative analyses of the optical density relative to the internal reference protein GAPDH in Western blot analysis. c Quantitative Real-time PCR analysis of ICAM-1 mRNA. Infection with P. gingivalis ATCC $33277(\mathrm{MOI}=100,24 \mathrm{~h})$ could induce the expression of ICAM-1 and ICAM-1 mRNA in EA.hy926 cells, this inductive effect of $P$. gingivalis was blocked by the MIF antagonist ISO-1. Sufficient exogenous recombinant MIF (rMIF) supplementation rescued the ICAM-1 expression. Data are presented as means \pm SD from three independent experiments. ${ }^{*} P<0.01$

\section{MIF regulates the increased monocyte adhesion to} endothelial cells infected with P. gingivalis ATCC 33277

To investigate the role of MIF in $P$. gingivalis-induced monocyte-endothelial cell adhesion, we used the fluorescent dye calcein-AM to highlight the adhesive THP-1 cells. THP-1 cell adhesion to EA.hy926 cells was visualized using fluorescence microscopy. The adhesion experiment results were consistent with ICAM-1 expression. Compared with uninfected cells, $P$. gingivalis ATCC 33277 infection (MOI = 100, 24 h) markedly increased THP-1 cell adhesion to endothelial cells $(P<$ $0.01)$. In contrast, cell adhesion was decreased in ISO-1treated cells compared with those infected with $P$. gingivalis ATCC $33277(P<0.01)$. In addition, THP-1 cell adhesion to EA.hy926 cells was recovered by exogenous rMIF addition, as shown in Fig. 3.

These results, in combination with those in Fig. 2, suggested that expression of ICAM-1 in endothelial cells and monocyte-endothelial cell adhesion caused by $P$. gingivalis ATCC 33277 infection could be regulated by MIF.

\section{Discussion}

Numerous cross-sectional, case-control and cohort epidemiological studies suggest that periodontal infection is associated with atherosclerotic CVD, independent of confounding factors such as smoking and obesity [25-27], and systemic inflammation has been proposed as a possible mediator [24], which ultimately enhances the adherence of circulating monocytes to vascular endothelial cells. Our prior study found that $P$. gingivalis infection induced ICAM-1 expression and monocyte recruitment, which are crucial events leading to atherosclerosis pathogenesis [20]. This result is consistent with the findings of Velsko [10]. P. gingivalis is believed to play a pivotal role in the development of atherosclerosis.

MIF is a proinflammatory cytokine that plays a critical role in the initiation and progression of chronic inflammatory and immune-mediated diseases such as atherosclerosis [28]. Under normal circumstances, the MIF protein level is very low. However, in atherosclerotic lesions, MIF is secreted in large quantities by vascular endothelial cells, and a relatively small amount of MIF is released by vascular smooth muscle cell [29]. Uniquely, MIF is rapidly released from preformed intracellular pools in response to LPS stimuli [30]. Consistently, in the current study, MIF secretion began to increase at $4 \mathrm{~h}$ after LPS stimulation, this increase sustained $24 \mathrm{~h}$. Li's research also confirmed there was a significantly higher level of MIF protein after stimulation with E. coli LPS for 24 h [21]. Interestingly, Li et al. also found that MIF protein level remained unchangeable in $P$. gingivalis LPS-treated reconstituted human gingival epithelia [21]. While our study showed that live $P$. gingivalis ATCC 


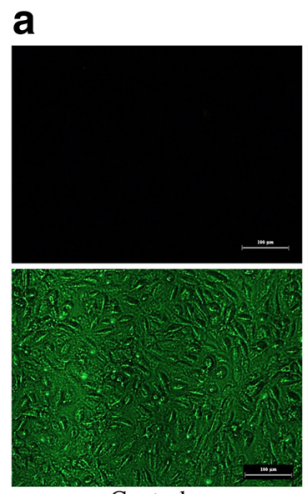

Control
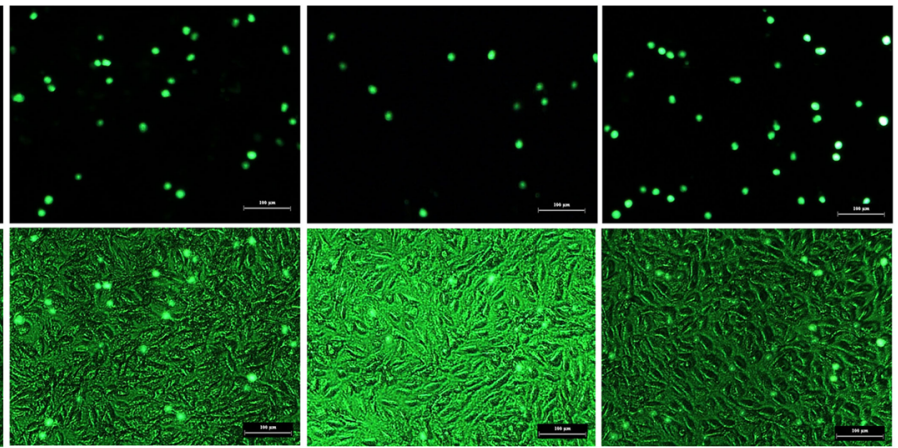

P.gingivalis

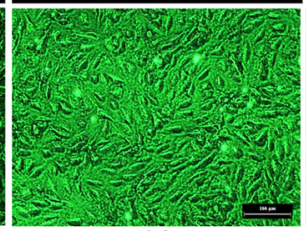

ISO-1

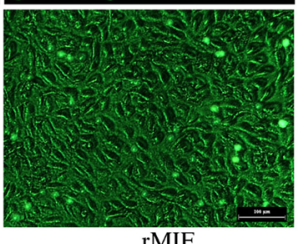

b

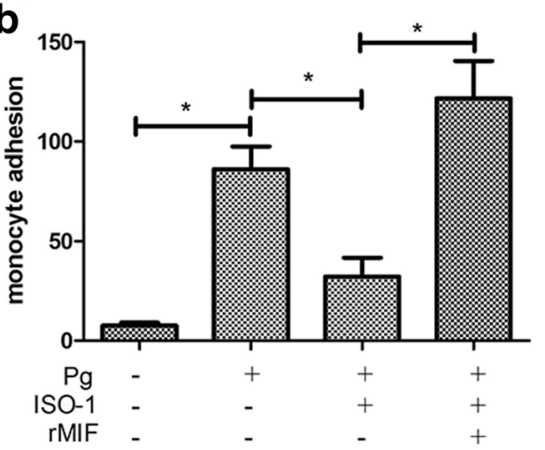

Fig. 3 Macrophage migration-inhibitory factor (MIF) regulated the increased monocyte adhesion to endothelial cells infected with Porphyromonas gingivalis ( $P$. gingivalis). a THP-1 cell adhesion to EA.hy926 cells was labeled by calcein-AM and visualized. Pictures are representative fields captured by flurencence microscope (upper line) or microscope (lower line) of three independent experiments (magnification $\times 100$ ). $\mathbf{b}$ The histogram of the evaluation of adhered THP-1 cell assessed by cell count assay. Compared with uninfected cells, P. gingivalis ATCC 33277 infection (MOI = 100, 24 h) markedly increased THP-1 cell adhesion to endothelial cells $(P<0.01)$. In contrast, cell adhesion was decreased in ISO-1-treated cells compared with those infected with $P$. gingivalis ATCC $33277(P<0.01)$. And THP-1 cell adhesion to EA.hy926 cells was recovered by exogenous rMIF addition. * $P<0.01$. Scale bar $=100 \mu \mathrm{m}$

33277-induced MIF secretion was weaker and much later than that induced by LPS. The results indicated that $P$. gingivalis ATCC 33277 had a different mechanism in inducing MIF expression compared to LPS. It has been reported that $P$. gingivalis can invade endothelial cells and remain viable for extended periods [31]. It is speculated that the invasion of $P$. gingivalis has significant repercussions for the physiological status of the cell.

Our findings identified a clue for the role of MIF in $P$. gingivalis-promoted atherosclerosis. Chuang's research showed that MIF induced by Dengue virus infection activates endothelial cell tight junction opening, which may cause plasma leakage and leukocyte migration (extravasation), resulting in increased vascular permeability [32]. Bernhagen et al. proved that MIF concentrations increase substantially in the presence of stress, inflammation, and infection [33]. We also noticed that the MIF concentration was increased in an unhealthy periodontal environment. MIF expression was higher in the periodontal tissue of chronic periodontitis patients than in that of healthy patients [21]. In experimental gingivitis patients, MIF protein expression in the gingival crevicular fluid started increasing 1 week after the occurrence of inflammation in the 46-77 years old age group. The trend in prostaglandin E2 expression is similar to that of MIF expression. According to statistical analyses, the MIF and PGE-2 concentrations are correlated, which suggests that MIF and PGE2 interact with each other have synergistically in inflammatory conditions [34].

The role of MIF in $P$. gingivalis infection was further investigated. MIF is a multifunctional cytokine with enzymatic tautomerase activity, and its inhibitor ISO-1 can block the activity of MIF [35]. We evaluated ICAM-1 expression by performing Western blot and qRT-PCR in endothelial cells infected with $P$. gingivalis for $24 \mathrm{~h}$ at an MOI of 100. Our prior work found that ICAM-1 expression and monocyte-endothelial cell adhesion were increased when endothelial cells were infected with $P$. gingivalis, which is consistent with the results of others $[36,37]$. In the presence of ISO-1, both the ICAM-1 protein and mRNA level induced by $P$. gingivalis infection were significantly decreased. However, ICAM-1 protein and mRNA expression levels were rescued by sufficient 
exogenous rMIF supplementation. We confirmed our results by cell adhesion assays. The endothelial cells were treated with ISO-1 or exogenous rMIF for $1 \mathrm{~h}$ before they were infected with $P$. gingivalis and then were co-cultured with monocytes. We found that monocyte adhesion to $P$. gingivalis ATCC 33277-infected endothelial cells was significantly inhibited by ISO-1. In contrast, sufficient rMIF supplementation retrieved the monocyte-endothelial cell adhesion. Recent evidence has suggested a role for endogenous MIF in the promotion of endothelial adhesion molecule expression [25]. Both Lin SG et al. [38] and Amin MA et al. [28] found that MIF up-regulated ICAM1 expression in endothelial cells. Moreover, in MIFdeficient human umbilical vein endothelial cells, the initial steps of atherosclerosis, such as the binding of adhesion molecules on endothelial cells to their specific ligands on mononuclear cells, or monocytes in circulation rolling and attaching to the vascular wall, were be accomplished due to a lack of extracellular MIF [15-17]. Our findings provide direct evidence for the role of MIF in upregulating ICAM-1 expression in $P$. gingivalis ATCC 33277-infected endothelial cells.

In summary, our study revealed that the MIF induced by $P$. gingivalis ATCC 33277 infection not only promoted ICAM-1 expression inendothelial cells but also activated monocyte-endothelial cell adhesion. We have shown that MIF is a very potent pathogenic factor in $P$. gingivalis ATCC 33277-induced atherosclerosis promotion. Suppressing MIF expression with an inhibitor or neutralizing antibody in individuals with manifest atherosclerosis may be a potential therapeutic intervention for treating this condition. However, the mechanisms whereby MIF facilitates endothelial adhesion molecule expression are unknown. Therefore, our future work will study the MIF receptor in $P$. gingivalis-infected endothelial cells.

\section{Conclusions}

The experiments revealed that endothelial cell-expressed MIF participates in pro-atherosclerotic lesion formation caused by $P$. gingivalis ATCC 33277 infection. Our novel findings elucidate a more detailed pathological role of $P$. gingivalis ATCC 33277 in atherosclerosis.

\footnotetext{
Abbreviations

CVD: Cardiovascular disease; DMEM: Dulbecco's modified Eagle medium; E. coli: Escherichia coli; ELISA: Enzyme linked immunosorbent assay; GAPDH: Glyceraldehyde-3-phosphatedehydrogenase; ICAM-1: Intercellular adhesion molecule - 1; LPS: Lipopolysaccharide; MIF: Macrophage migration inhibitory factor; MOI: Multiplicity of infection; P. gingivalis: Porphyromonas gingivalis; qRT-PCR: Quantitative real-time polymerase chain reaction; rMIF: recombinant MIF
}

Acknowledgements

Not applicable.

\section{Funding}

This study was supported by grants from National Natural Science Foundation of China (No. 81400518, 81670997, 81500869). The funders had no role in study design, data collection and analysis, or preparation of the manuscript.

\section{Availability of data and materials}

The datasets used and/or analysed during the current study are available from the corresponding author on reasonable request.

\section{Authors' contributions}

Conception and design of study: ZD, PY. Cell culture, ELISA and real-time PCR assay: XW, XQ, WY. Bacterial culture and western blot analysis: XW, PJ, HJ. Acquisition of data: LC, LJ. Analysis and interpretation: PY, ZD, LL, TX. Writing the manuscript: $Z D, P Y, X W$. All authors read and approved the final manuscript.

Ethics approval and consent to participate

Not applicable.

\section{Consent for publication}

Not applicable.

\section{Competing interests}

The authors declare that they have no competing interests.

\section{Publisher's Note}

Springer Nature remains neutral with regard to jurisdictional claims in published maps and institutional affiliations.

Received: 25 August 2017 Accepted: 8 February 2018

Published online: 26 February 2018

\section{References}

1. Zhang B, Khalaf $H$, Sirsjö A, Bengtsson T. Gingipains from the periodontal pathogen Porphyromonas gingivalis play a significant role in regulation of angiopoietin 1 and angiopoietin 2 in human aortic smooth muscle cell. Infect Immun. 2015;83:4256-65.

2. Kebschull M, Demmer RT, Papapanou PN. "Gum bug, leave my heart alone!"-epidemiologic and mechanistic evidence linking periodontal infections and atherosclerosis. J Dent Res. 2010;89:879-902.

3. Cavrini F, Sambri V, Moter A, Servidio D, Marangoni A, Montebugnoli L, et al Molecular detection of Treponema denticola and Porphyromonas gingivalis in carotid and aortic atheromatous plaques by FISH: report of two cases. J Med Microbiol. 2005;54:93-6.

4. Haraszthy VI, Zambon JJ, Trevisan M, Zeid M, Genco RJ. Identification of periodontal pathogens in atheromatous plaques. J Periodontol. 2000;71: 1554-60.

5. Koizumi Y, Kurita-Ochiai T, Oguchi S, Yamamoto M. Nasal immunization with Porphyromonas gingivalis outer membrane protein decreases $P$. gingivalis-induced atherosclerosis and inflammation in spontaneously hyperlipidemic mice. Infect Immun. 2008;76:2958-65.

6. Brodala N, Merricks EP, Bellinger DA, Damrongsri D, Offenbacher S, Beck J, et al. Porphyromonas gingivalis bacteremia induces coronary and aortic atherosclerosis in normocholesterolemic and hypercholesterolemic pigs. Arterioscler Thromb Vasc Biol. 2005;25:1446-51.

7. Rivera MF, Lee JY, Aneja M, Goswami V, Liu L, Velsko IM, et al. Polymicrobial infection with major periodontal pathogens induced periodontal disease and aortic atherosclerosis in Hyperlipidemic ApoE(null) mice. PLoS One. 2013;8:e57178

8. Velsko IM, Chukkapalli SS, Rivera MF, Lee JY, Chen H, Zheng D, et al. Active invasion of oral and aortic tissues by Porphyromonas gingivalis in mice causally links periodontitis and atherosclerosis. PLoS One. 2014;9:e97811.

9. Chukkapalli SS, Rivera MF, Velsko IM, Lee JY, Chen H, Zheng D, et al. Invasion of oral and aortic tissues by oral spirochete Treponema denticola in $\mathrm{ApoE}(-/-)$ mice causally links periodontal disease and atherosclerosis. Infect Immun. 2014;82:1959-567.

10. Chukkapalli SS, Rivera-Kweh MF, Velsko IM, Chen H, Zheng D, Bhattacharyya I, Gangula PR, Lucas AR, Kesavalu L, et al. Chronic oral infection with major periodontal bacteria Tannerella forsythia modulates systemic atherosclerosis risk factors and inflammatory markers. Patho Dis. 2015;73:ftv009. 
11. Velsko IM, Chukkapalli SS, Rivera-Kweh MF, Zheng D, Aukhil I, Lucas AR, et al. Periodontal pathogens invade gingiva and aortic adventitia and elicit inflammasome activation in avß6 integrin-deficient mice. Infect Immun. 2015:83:4582-93.

12. Zernecke A, Bernhagen J, Weber C. Macrophage migration inhibitory factor in cardiovascular disease. Circulation. 2008;117:1594-602.

13. Chen D, Xia M, Hayford C, Tham e-L, Semik V, Hurst S, et al. Expression of human tissue factor pathway inhibitor on vascular smooth muscle cells inhibits secretion of macrophage migration inhibitory factor and attenuates atherosclerosis in ApoE-/- mice. Circulation. 2015;131:1350-60.

14. Sun H, Zhang X, Zhao L, Zhen X, Huang S, Wang S, et al. Attenuation of atherosclerotic lesions in diabetic apolipoprotein E-deficient mice using gene silencing of macrophage migration inhibitory factor. J Cell Mol Med. 2015;19:836-49.

15. Morand EF, Leech M, Bernhagen J. MIF: a new cytokine link between rheumatoid arthritis and atherosclerosis. Nat Rev Drug Discov. 2006;5:399-410.

16. Gong Z, Xing S, Zheng F, Xing Q. Increased expression of macrophage migration inhibitory factor in aorta of patients with coronary atherosclerosis. J Cardiovasc Surg. 2015;56:631-7.

17. Asare Y, Schmitt M, Bernhagen J. The vascular biology of macrophage migration inhibitory factor (MIF). Expression and effects in inflammation, atherogenesis and angiogenesis. Thromb Haemost. 2013;109:391-8.

18. Zhou B, Ren C, Zu L, Zheng L, Guo L, Gao W. Elevated plasma migration inhititory factor in hypertension-hyperlipdemia patients correlates with impaired endothelial function. Medicine (Baltimore). 2016;95:e5207.

19. Blankenberg S, Barbaux S, Tiret L. Adhesion molecules and atherosclerosis. Atherosclerosis. 2003;170:191-203.

20. Zhang D, Zheng H, Zhao J, Lin L, Li C, Liu J, et al. Porphorymonas gingivitis induces intracellular adhesion molecule-1 expression in endothelial cells through the nuclear factor-kappaB pathway, but not through the p38 MAPK pathway. J Periodontal Res 2011;46:31-38.

21. Li X, Lan HY, Huang XR, Zhang C, Jin LJ. Expression profile of macrophage migration-inhibitory factor in human gingiva and reconstituted human gingival epithelia stimulated by Porphyromonas gingivalis lipopolysaccharide. J Periodontal Res. 2013;48(4):527-32.

22. Meng W, Hong Y, Feng K, Deng P, Song W, Nie X. Genome-wide Identification, phylogeny and expressional profiles of mitogen activated protein kinase kinase kinase (MAPKKK) gene family in bread wheat (Triticum aestivumL). BMC Genomics, 2016, 17(1):668-689.

23. Hsuan CF, Hsu HF, Tseng WK, Lee TL, Wei YF, Hsu KL, et al. Glossogyne Tenuifolia extract inhibits TNF-a-induced expression of adhesion molecules in human umbilical vein endothelial cells via blocking the NF-kB signaling pathway. Molecules. 2015;20:16908-23.

24. Calandra T, Roger T. Macrophage migration inhibitory factor: a regulator of innate immunity. Nat Rev Immunol. 2003;3(10):791-800.

25. Hajishengallis G. Periodontitis: from microbial immune subversion to systemic inflammation. Nat Rev Immunol. 2015;15:30-44.

26. Bahekar AA, Singh S, Saha S, Molnar J, Arora R. The prevalence and incidence of coronary heart disease is significantly increased in periodontitis: a meta-analysis. Am Heart J. 2007;154:830-7.

27. Friedewald VE, Kornman KS, Beck JD, Genco R, Goldfine A, Libby P, et al. The American journal of cardiology and journal of periodontology Editors' consensus: periodontitis and atherosclerotic cardiovascular disease. Am J Cardiol. 2009;104:59-68.

28. Amin MA, Haas CS, Zhu K, Mansfield PJ, Kim MJ, Lackowski NP, et al. Migration inhibitory factor up-regulates vascular cell adhesion molecule-1 and intercellular adhesion molecule-1 via Src, PI3 kinase, and NF-kappaB. Blood. 2006;107:2252-61.

29. White DA, Su Y, Kanellakis P, Kiriazis H, Morand EF, Bucala R, et al. Diffrential roles of cardiac and leukocyte derived macrophage migration inhibitory factor in inflmmatory responses and cardiac remodelling post myocardial infarction. J Mol Cell Cardiol. 2014;69:32-42.

30. Finucane OM, Reynolds CM, McGillicuddy FC, Roche HM. Insights into the role of macrophage migration inhibitory factor in obesity and insulin resistance. Proc Nutr Soc. 2012;71(4):622-33.

31. Lamont RJ, Yilmaz O. In or out: the invasiveness of oral bacteria. Periodontol 2000. 2002:30:61-9.

32. Chuang YC, Lei HY, Liu HS, Lin YS, Fu TF, Yeh TM. Macrophage migration inhibitory factor induced by dengue virus infection increases vascular permeability. Cytokine. 2011:54:222-31.
33. Bernhagen J, Krohn R, Lue H, Gregory JL, Zernecke A, Koenen RR, et al. MIF is a noncognate ligand of CXC chemokine receptors in inflammatory and atherogenic cell recruitment. Nat Med. 2007;13:587-96.

34. Nonnenmacher $C$, Helms $K$, Bacher $M$, Nüsing RM, Susin C, Mutters $R$, et al. Effect of age on gingival crevicular fluid concentrations of MIF and PGE2. J Dent Res. 2009;88:639-43.

35. Leng L, Bucala R. Macrophage migration inhibitory factor. Crit Care Med. 2005;33:S475-7.

36. Hirose $K$, Isogai $E$, Ueda I. Porphyromonas gingivalis fimbriae induce adhesion of monocytic cell line U937 to endothelial cells. Microbiol Immunol. 2000;44:17-22

37. Hashizume T, Kurita-Ochiai T, Yamamoto M. Porphyromonas gingivalis stimulates monocyte adhesion to human umbilical vein endothelial cells. FEMS Immunol Med Microbiol. 2011;62:57-65.

38. Lin SG, Yu XY, Chen YX, Huang XR, Metz C, Bucala R, et al. De novo expression of macrophage migration inhibitory factor in atherogenesis in rabbits. Circ Res. 2000:87:1202-8.

\section{Submit your next manuscript to BioMed Central and we will help you at every step:}

- We accept pre-submission inquiries

- Our selector tool helps you to find the most relevant journal

- We provide round the clock customer support

- Convenient online submission

- Thorough peer review

- Inclusion in PubMed and all major indexing services

- Maximum visibility for your research

Submit your manuscript at www.biomedcentral.com/submit

) Biomed Central 